\title{
La publicité directe au consommateur : fini les compromis
}

$\mathrm{M}$ ême si les règlements fédéraux interdisent la publicité directe au consommateur pour les médicaments et les instruments médicaux, elle est devenue courante au Canada. Dans les pages de People, Ladies' Home Journal, Redbook et autres publications américaines, on peut apprendre comment protéger sa jeune fille contre les ravages du VPH, se protéger soi-même contre un infarctus du myocarde à venir en prenant Plavix ou Crestor, ou minimiser la destruction des articulations et la douleur causées par la polyarthrite rhumatoïde en utilisant de nouveaux anticorps monoclonaux — tout en attendant de payer l'épicerie à la caisse. On peut aussi entendre les sociétés pharmaceutiques parler de leurs nouveaux traitements en regardant des reprises de Friends ou de Law and Order sur les grandes chaînes de télévision américaines retransmises au Canada par satellite ou par les câblodistributeurs.

La publicité directe au consommateur semble interdite seulement pour les médias et les entreprises du Canada ${ }^{1}$. Suivant une façon bizarre d'appliquer ses règlements, Santé Canada permet les annonces sibyllines qui désignent nommément le problème médical ou le produit, mais non les 2 . Les sociétés américaines, elles, annoncent impunément au Canada. Elles fonctionnent en fait au Canada en suivant les règles américaines. Aux États-Unis, les annonces sont réglementées par l'industrie et contrôlées a posteriori seulement par la US Food and Drug Administration (FDA). La FDA écrit aux entreprises qui violent les règles - la plupart du temps parce que leur publicité exagère l'efficacité et minimise le risque ${ }^{2}$.

À l'échelle mondiale, seuls les États-Unis et la Nouvelle-Zélande permettent la publicité directe au consommateur pour les médicaments. Ailleurs, les lois visent à garantir que les patients vulnérables ne sont pas la cible d'affirmations partielles ou embellies des fabricants de médicaments. La façon de procéder du Canada est manifestement bizarre. On n'applique pas les règlements, on ne filtre pas les annonces et Santé Canada fait la sourde oreille à la publicité américaine sur les médicaments qui s'adresse au consommateur. C'est en fait ce qui permet de bombarder les Canadiens d'annonces de produits pharmaceutiques américains alors qu'il est interdit aux entreprises canadiennes de diffuser des annonces semblables.

Cette double norme n'a à notre avis aucun sens et il se peut fort bien que les tribunaux soient d'accord.

CanWest Global Media, un des plus importants conglomérats médiatiques du Canada, poursuit le gouvernement canadien en affirmant que les lois et règlements actuels enfreignent son droit à la libre expression enchâssé dans la Charte canadienne des droits et libertés. L'inaction de Santé Canada, qui permet la publicité directe au consommateur — à condition qu'elle provienne d'ailleurs — pourrait donner plus de poids à son argument, ce qui est ironique.

Les arguments contre la publicité directe au consommateur pour les médicaments d'ordonnance se fondent sur un impératif de sécurité publique ${ }^{3}$. Les campagnes publicitaires entraînent une augmentation de la demande de médicaments annoncés et du nombre des ordonnances. Souvent, ces nouveaux médicaments coûtent plus cher et on ne comprend pas bien leur profil risqueavantage. Quelque $20 \%$ des nouveaux médicaments finissent par faire l'objet d'un avertissement de sécuritét. En effet, les médicaments et les instruments sont évalués au début dans des circon- stances idéales avec un nombre limité de patients. Dans les conditions habituelles de soin, les risques sont souvent plus grands et les avantages, moins nombreux.

Les promoteurs de la publicité sur les médicaments qui s'adresse au consommateur préconisent une meilleure information du consommateur en matière de santé et affirment que ces annonces font connaître au patient bien informé les solutions de rechange qui s'offrent à lui ${ }^{5}$. N'oublions pas, toutefois, qu'un des grands buts de l'industrie pharmaceutique, c'est d'augmenter la valeur pour l'actionnaire. Il s'ensuit que les campagnes de publicité ne seront jamais entièrement impartiales, en fait ou en apparence. L'information du public sur les risques et les avantages de certaines interventions en santé peut être une retombée secondaire de la publicité adressée au consommateur, mais la partialité demeure.

L'éducation des patients relève de la compétence des médecins et autres professionnels de la santé, qui doivent présenter des recommandations factuelles. Pour appuyer les patients et les professionnels de la santé dans leurs décisions, nous avons besoin de sources accessibles d'information impartiale au sujet des instruments et des médicaments, et non d'autres campagnes de publicité.

Dans l'intérêt de la justice et de la santé publique, Santé Canada doit appliquer de façon uniforme les règlements en vigueur sur la publicité directe au consommateur, peu importe le pays d'origine. $\mathrm{Si}$ Santé Canada ne fait rien, les tribunaux pourraient très bien prendre la décision pour nous et lever l'interdiction de la publicité directe au consommateur pour les médicaments d'origine canadienne. Sur le mode du compromis si typiquement canadien, il se pourrait fort bien que l'on en vienne à sacrifier la santé publique et la sécurité des consommateurs en faveur de l'égalité des chances.

\section{Paul C. Hébert MD MHSc}

Rédacteur en chef

$J A M C$

Avec l'équipe de rédaction de l'éditorial (Rajendra Kale MD,

Matthew B. Stanbrook MD PhD, Barbara Sibbald BJ,

Ken Flegel MDCM MSc, Noni MacDonald MD MSc, Amir Attaran LLB DPhil)

Intérêts concurrents : Voir www.cmaj.ca/misc/edboard.shtml.

Traduit par le Service de traduction de l'AMC.

\section{RÉFÉRENCES}

1. Loi sur les aliments et drogues : loi concernant les aliments, drogues, cosmétiques et instruments thérapeutiques. LR (1985) c F-27. Disponible : http://laws.justice.gc.ca /fr/ShowFullDoc/cs/F-27/bo-ga:s_1: bo-ga:s_2//fr?page=1 (consulté le 12 juin 2008).

2. Mintzes B. Publicité directe aux consommateurs des médicaments d'ordonnance au Canada: Quelles en sont les conséquences sur la santé publique? Toronto (Ont.) : Conseil canadien de la santé; 2006.

3. Mintzes B, Barer ML, Kravitz RL, et al. How does direct-to-consumer advertising (DTCA) affect prescribing? A survey in primary care environments with and without legal DTCA. JAMC 2003;169:405-14.

4. Lasser KE, Allen PD, Woolandler SJ, et al. Timing of new black box warnings and withdrawals for prescription medications. JAMA 2002;287:2215-20.

5. Aikin KJ, Swasy JL, Braman AC. Patient and physician attitudes and behaviors associated with DTC promotion of prescription drugs - summary of FDA survey research results: final report. Beltsville (MD): FDA Center for Drug Evaluation and Research; 2004 